\title{
Irradiance Estimation Using Kalman Filter for State Space Systems with Unknown Inputs
}

\author{
Vinícius Souza Madureira ${ }^{*, * *}$ Thiago Pereira das Chagas ${ }^{*, * *}$ \\ Gildson Queiroz de Jesus ${ }^{* * *}$ \\ * Programa de Pós-Graduação em Modelagem Computacional em \\ Ciência e Tecnologia, Universidade Estadual de Santa Cruz, \\ Ilhéus-Bahia,Brasil (e-mails: vinicius.madu@gmail.com, \\ tpchagas@uesc.br,gildsonj@gmail.com) \\ ** Laboratório de Mecatrônica, Departamento de Ciências Exatas e \\ Tecnológicas, Universidade Estadual de Santa Cruz, Ilhéus-BA, Brasil
}

\begin{abstract}
:
This work presents a method for solar irradiance estimation based on Kalman filter for systems subject to unknown inputs. A system composed of a photovoltaic panel, a dc-dc converter and a load was modeled as state space subject to unknown input. The results of the simulated case studies presents a normalized root mean square error (nRMSE) between $0.93 \%$ and $14.23 \%$ for irradiance estimates, the latter value corresponding to a case that considers adverse situations in applications. In addition, the proposed methodology also estimates the output voltage (nRMSE between $5.66 \%$ and $13.15 \%$ ) and output current (nRMSE between $0.65 \%$ and $12.46 \%$ ) of photovoltaic panels. All these estimates are based on the measurement of a single voltage sensor at the dc-dc converter output. Thus, the KFUI based irradiance estimation is a low cost alternative to the use of pyranometers and has good perspectives for new works on filtering and control of photovoltaic systems.
\end{abstract}

Keywords: photovoltaic systems, irradiance estimation, Kalman filter, unknown inputs, boost converter

\section{INTRODUCTION}

In order to know the solar energy generation potential in a given location, accurate information is needed regarding the available solar resource (Martins and Pereira, 2011). Such information is also important for the management of the solar systems in operation since it is used in applications such as Maximum Power Point Tracking (MPPT) (Chikh and Chandra, 2015; Scolari et al., 2017) and control of hybrid power generation systems (Mohamed and Mohammed, 2013). However, the solar resource presents high variability as it is associated with the Earth's rotation and translation movement and depends on local meteorological factors (Pereira et al., 2017).

The most reliable way to measure solar irradiance is through pyranometers (Martins and Pereira, 2011). However, this sensor requires periodic calibrations and, depending on the photovoltaic system, can be expensive. In this way, recent researches seeks cheaper alternatives by estimating irradiance rather than measuring it directly. The state-of-the-art is strongly linked to the use of satellites (Miller et al., 2018), but other proposals also uses image processing (Scolari et al., 2018) or the mathematical model resulting from the equivalent circuit of the photovoltaic panel along with current and voltage sensors (Scolari et al., 2017; Chikh and Chandra, 2015; Moshksar and Ghanbari, 2018).
In this work is proposed to use the equivalent circuit known as single diode together with the state space model of dc-dc converter, which is a device commonly used in photovoltaic systems. By combining these two models results in a state space model in which the input is the output voltage of the photovoltaic panel. As it is directly associated with solar irradiance, this input also presents great variability and therefore is treated as an unknown input (Darouach et al., 1995).

From the obtained state space model, a wide variety of filtering and control applications for photovoltaic systems can be approached. For example, strategies to perform MPPT, control hybrid systems of generation, estimate variables using Kalman filter, among others.

In some situations, the state variables of a system can be estimated through other measures by standard Kalman filter (KF). As irradiance is expensive to measure in a large photovoltaic system, where a lot of pyranometers can be needed, the $\mathrm{KF}$ is an alternative to estimate it. However, because of irradiance unknown input nature, the Kalman filter approach for state space systems subject to unknown inputs (KFUI) as proposed by Darouach et al. (1995) is used in this work to estimate irradiance.

The advantage of the proposed methodology is to require only one sensor to measure the output voltage of dc-dc converter. The other measurements (photovoltaic panel 
output voltage and current) are estimated by the KFUI. Other proposals that use the single diode model require both voltage and current measurements (Scolari et al., 2017; Moshksar and Ghanbari, 2018).

Thus, this work contributes to a low cost alternative in which only dc-dc converters and KFUI are required to estimate irradiance, current and voltage in photovoltaic panels.

\section{SINGLE DIODE PHOTOVOLTAIC PANEL MODEL}

The single diode circuit of a photovoltaic panel, which has $N_{S}$ cells arranged in series and $N_{P}$ panels in parallel, is shown in Figure 1 (Nguyen and Nguyen, 2015).

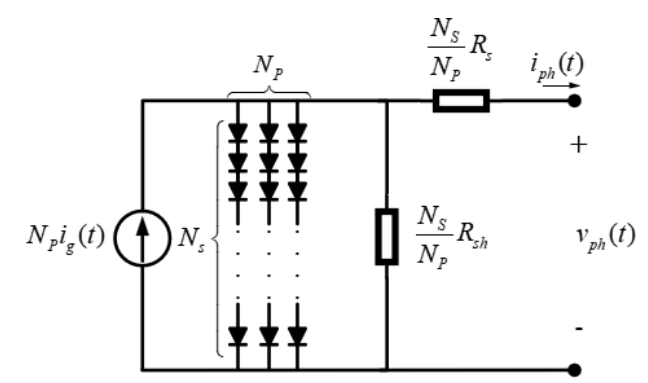

Figure 1. Equivalent circuit of a photovoltaic panel

At each instant of time $t$, the generated current $i_{g}(t)$ is affected by temperature $T(t)$ and irradiance $G(t)$ according to (1) (Nguyen and Nguyen, 2015),

$$
i_{g}(t)=\frac{G(t)}{G_{r}}\left[I_{s c}+\alpha_{I_{s} c}\left(T(t)-T_{r}\right)\right]
$$

where $G_{r}$ is the reference solar irradiance, generally 1000 $\mathrm{W} / \mathrm{m}^{2} ; T_{r}$ is the reference temperature, $298 \mathrm{~K} ; I_{s c}$ is the short-circuit current of the photovoltaic cell at the reference conditions $\left(1000 \mathrm{~W} / \mathrm{m}^{2}\right.$ and $\left.298 \mathrm{~K}\right)$; and $\alpha_{I_{s} c}$ is the temperature coefficient of the short-circuit current given in $\mathrm{A} /{ }^{\circ} \mathrm{C}$.

Equation (2) can be used to calculate the current $i_{p h}(t)$ supplied by a photovoltaic (Nguyen and Nguyen, 2015),

$$
i_{p h}(t)=N_{P} i_{g}(t)-N_{P} i_{d}(t)-\frac{N_{P}}{R s h}\left(\frac{v_{p h}(t)}{N_{S}}+\frac{i_{p h}(t) R_{S}}{N_{P}}\right),
$$

where $i_{d}(t)$, given by (3), is the current which flows through each series set of diodes,

$$
i_{d}(t)=I_{0}(t)\left[\exp \left(\frac{q\left(\frac{v_{p h}(t)}{N_{s}}+\frac{i_{p h}(t) R_{s}}{N_{P}}\right)}{a B T_{r}}\right)-1\right]
$$

where $q$ is the charge of an electron, $1.60 \times 10^{-3} \mathrm{C} ; a$ is the ideality factor of the diode; $B$ is Boltzmann's constant, $1.38 \times 10^{-38} \mathrm{~J} / \mathrm{K}$; and $I_{0}(t)$ is the reverse saturation current of the diode, given by (4) (Nguyen and Nguyen, 2015),

$$
I_{0}(t)=I_{0 r}\left(\frac{T(t)}{T_{r}}\right)^{3} \exp \left[\frac{q V_{g}}{a B}\left(\frac{1}{T_{r}}-\frac{1}{T(t)}\right)\right],
$$

where $V_{g}$ is the bandgap voltage and $I_{0 r}$ is the reverse saturation current of the diode in the reference conditions given by (5) (Nguyen and Nguyen, 2015),

$$
I_{0 r}=\frac{I_{s c}}{\exp \left(\frac{q V_{o c}}{a B T_{r} N_{S}}\right)-1}
$$

where $V_{o c}$ is the open circuit voltage of the panel.

An analytical form to calculate irradiance is obtained substituting (1) in (2) and solving for $G(t)$, according to (6),

$$
\begin{aligned}
G(t)= & {\left[\frac{i_{p h}(t)}{N_{P}}+i_{d}(t)+\frac{1}{R s h}\left(\frac{v_{p h}(t)}{N_{S}}+\frac{i_{p h}(t) R_{s}}{N_{P}}\right)\right] \times } \\
& \frac{G_{r}}{I_{s c}+\alpha_{I_{s} c}\left(T(t)-T_{r}\right)}
\end{aligned}
$$

Manufacturer's datasheet of the photovoltaic panel provides the constants $V_{o c}, I_{s c}, \alpha I_{s c}$ and $N_{S}$. As shown in Tian et al. (2012), the values of $R_{s}, R_{s h}, n$ and $V_{g}$ can be calculated. Since temperature can be easily measured, in this work it will be considered constant at $25^{\mathrm{O}} \mathrm{C}$. Thus, calculation of $G(t)$ requires the knowledge of two variables, $i_{p h}(t)$ and $v_{p h}(t)$.

\section{BOOST CONVERTER MODEL}

As mentioned previously, this work provides a methodology for photovoltaic generation systems with a conversion stage. A boost converter was used, but the methodology can be similarly applied to other types of converters.

Figure 2 shows the circuit with the photovoltaic panel and converter connected. The output voltage $v_{p h}(t)$ and current $i_{p h}(t)$ of the photovoltaic panel are the input

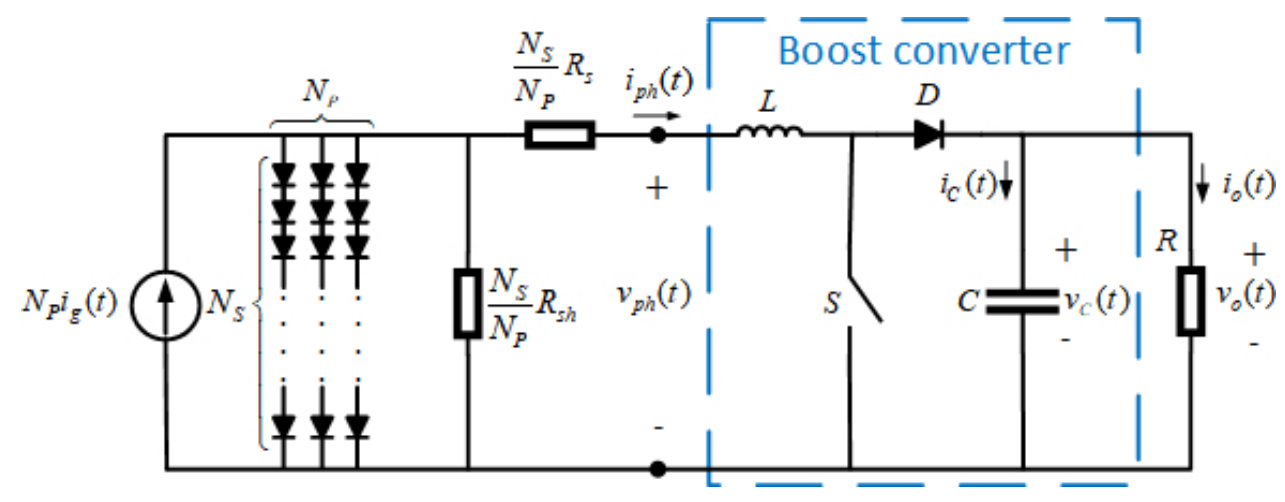

Figure 2. Photovoltaic panel connected to boost converter 
voltage and current of the converter, respectively. Thus, the variations of irradiance also affects the output voltage $v_{o}(t)$ of the converter.

Considering a duty cycle $d(t)$, the linearized model of boost converter is presented in (7) (Erickson and Maksimović, 2001),

$$
\begin{aligned}
& L \frac{d}{d t} \tilde{i}_{p h}(t)=\tilde{v}_{p h}(t)-(1-D) \tilde{v}_{o}(t)+V_{o} \tilde{d}(t), \\
& C \frac{d}{d t} \tilde{v}_{o}(t)=(1-D) \tilde{i}_{p h}(t)-I_{p h} \tilde{d}(t)-\frac{\tilde{v}_{o}(t)}{R} .
\end{aligned}
$$

This model is provided by a small signal analysis of the converter. Since the signals $i_{p h}(t), v_{o}(t), v_{p h}(t)$ and $d(t)$ oscillate around a quiescent point, is possible to rewrite them as the sums presented in (8) to (11) (Erickson and Maksimović, 2001),

$$
\begin{gathered}
i_{p h}(t)=I_{p h}+\tilde{i}_{p h}(t), \\
v_{o}(t)=V_{o}+\tilde{v}_{o}(t), \\
v_{p h}(t)=V_{p h}+\tilde{v}_{p h}(t), \\
d(t)=D+\tilde{d}(t),
\end{gathered}
$$

where $I_{p h}, V_{o}, V_{p h}$ and $D$ are the quiescent operating points of $i_{p h}(t), v_{o}(t), v_{p h}(t)$ and $d(t)$, respectively; and $\tilde{i}_{p h}(t)$, $\tilde{v}_{o}(t), \tilde{v}_{p h}(t)$ and $\tilde{d}(t)$ are small variations around these quiescent values.

In the state space form and considering $y(t)=v_{o}(t)$ the measured variable, the equation $(7)$ is a linear and time invariant system, as presented in (12),

$$
\begin{aligned}
\dot{\tilde{\mathbf{x}}}(t)=\mathcal{F} \tilde{\mathbf{x}}(t) & +\mathcal{G} \tilde{d}(t)+\mathcal{E} \tilde{v}_{p h}(t) \\
\tilde{y}(t) & =\mathcal{H} \tilde{\mathbf{x}}(t),
\end{aligned}
$$

where

$$
\mathcal{F}=\left[\begin{array}{cc}
0 & -\frac{(1-D)}{L} \\
\frac{(1-D)}{C} & -\frac{1}{R C}
\end{array}\right] \text { is the system parameter ma- }
$$

trix, $\mathcal{G}=\left[\frac{V_{0}}{L}-\frac{I_{p h}}{C}\right]^{T}$ is the parameter vector of input $\tilde{d}(t), \mathcal{E}=\left[\frac{1}{L} 0\right]^{T}$ is the parameter vector of input $\tilde{v}_{p h}(t), \mathcal{H}=\left[\begin{array}{ll}0 & 1\end{array}\right]$ is the output parameter vector and $\tilde{\mathbf{x}}(t)=\left[\tilde{i}_{p h}(t) \tilde{v}_{o}(t)\right]^{T}$ is the system state.

Values of $I_{p h}, V_{o}, V_{p h}, D, L, R$ and $C$ are known from the design of the converter.

The value of $v_{o}(t)$ can be controlled by adjusting the input $\tilde{d}(t)$ through the switching signal of the converter. The voltage $\tilde{v}_{p h}(t)$ is supplied by the photovoltaic panel and thus depends directly on solar irradiance, which is a random and non manipulable variable. Thus, it can be said that $\tilde{v}_{p h}(t)$ is an unknown input to the system of (12) (Darouach et al., 1995).

\section{KALMAN FILTER FOR SYSTEMS WITH UNKNOWN INPUTS (KFUI)}

Given the state space model according to (12), this work proposes to estimate the values of $\tilde{i}_{p h}(t)$ and $\tilde{v}_{p h}(t)$ through
KFUI (Darouach et al., 1995). Consequently, irradiance can be estimated using (6).

Consider the discrete state space system represented by (13),

$$
\begin{gathered}
\mathbf{x}_{k+1}=\mathbf{F} \mathbf{x}_{k}+\mathbf{G} \mathbf{u}_{k}+\mathbf{E} \mathbf{z}_{k}+\mathbf{w}_{k} \\
\mathbf{y}_{k+1}=\mathbf{H} \mathbf{x}_{k+1}+\mathbf{v}_{k},
\end{gathered}
$$

where $\mathbf{x}_{k} \in \mathbb{R}^{n}$ is the state vector; $\mathbf{u}_{k} \in \mathbb{R}^{m}$ is the input or control vector; $\mathbf{z}_{k} \in \mathbb{R}^{p}$ is the unknown input vector; $\mathbf{y}_{k+1} \in \mathbb{R}^{q}$ is the output vector; $\mathbf{w}_{k} \in \mathbb{R}^{n}$ and $\mathbf{v}_{k} \in \mathbb{R}^{q}$ are white noise vectors with zero mean, uncorrelated with each other and with the initial state of the system and with covariates $\mathbf{W}(n \times n)$ and $\mathbf{V}(q \times q)$, respectively; $\mathbf{F}$ is the $(n \times n)$ system parameter matrix; $\mathbf{G}$ is the $(n \times m)$ input parameter matrix; $\mathbf{E}$ is the $(n \times p)$ parameter matrix of the unknown inputs; $\mathbf{H}$ is the $(q \times n)$ output parameter matrix; and $k=0,1,2 \ldots, N$ is a sequence of integers for an amount $N$ of available measures.

The optimal estimates of the unknown input and the state are given by (14) to (16) (Darouach et al., 1995),

$$
\begin{gathered}
\overline{\mathbf{x}}_{k \mid k}=\mathbf{F} \hat{\mathbf{x}}_{k \mid k}+\mathbf{G} \mathbf{u}_{k}, \\
\hat{\mathbf{x}}_{k+1 \mid k+1}=\overline{\mathbf{x}}_{k \mid k}+\mathbf{E} \hat{\mathbf{z}}_{k \mid k+1}+ \\
\mathbf{K}_{k+1}^{x}\left[\mathbf{y}_{k+1}-\mathbf{H}\left(\overline{\mathbf{x}}_{\mathbf{k} \mid \mathbf{k}}+\mathbf{E} \hat{\mathbf{z}}_{k \mid k+1}\right)\right] \\
\hat{\mathbf{z}}_{k \mid k+1}=\mathbf{K}_{k+1}^{z}\left(\mathbf{y}_{k+1}-\mathbf{H} \overline{\mathbf{x}}_{k \mid k}\right),
\end{gathered}
$$

where $\hat{\mathbf{x}}$ and $\hat{\mathbf{z}}$ are the estimates of system state and unknown inputs, respectively. $\mathbf{K}_{k+1}^{x}$ and $\mathbf{K}_{k+1}^{z}$ are given by (17) and (18),

$$
\begin{gathered}
\mathbf{K}_{k+1}^{x}=\left(\overline{\mathbf{P}}_{k \mid k}^{-1}+\mathbf{H}^{T} \mathbf{V}^{-1} \mathbf{H}\right)^{-1} \mathbf{H}^{T} \mathbf{V}^{-1} \\
\mathbf{K}_{k+1}^{z}=\mathbf{P}_{k+1 \mid k+1}^{z x} \mathbf{H}^{T} \mathbf{V}^{-1}
\end{gathered}
$$

with

$$
\begin{gathered}
\overline{\mathbf{P}}_{k \mid k}=\mathbf{F P}_{k \mid k}^{x} \mathbf{F}^{T}+\mathbf{W}, \\
\mathbf{P}_{k+1 \mid k+1}^{z x}=\mathbf{P}_{k \mid k+1}^{z} \mathbf{E}^{T} \overline{\mathbf{P}}_{k \mid k}^{-1}\left(\overline{\mathbf{P}}_{k \mid k}^{-1}+\mathbf{H}^{T} \mathbf{V}^{-1} \mathbf{H}\right)^{-1}, \\
\mathbf{P}_{k \mid k+1}^{z}=\left(\mathbf{E}^{T} \mathbf{H}^{T}\left(\mathbf{V}+\mathbf{H}_{k \mid k} \mathbf{H}^{T}\right)^{-1} \mathbf{H E}\right)^{-1}, \\
\mathbf{P}_{k+1 \mid k+1}^{x}=\left(\overline{\mathbf{P}}_{k \mid k}^{-1}+\mathbf{H}^{T} \mathbf{V}^{-1} \mathbf{H}\right)^{-1}+ \\
\mathbf{P}_{k+1 \mid k+1}^{x z}\left(\mathbf{P}_{k \mid k+1}^{z}\right)^{-1} \mathbf{P}_{k+1 \mid k+1}^{z x},
\end{gathered}
$$

in which $\mathbf{V}$ and $\mathbf{W}$ are weighting matrices corresponding to unknown covariances, $\mathbf{P}^{x}$ is the $(n \times n)$ covariance matrix of the state estimation error, $\mathbf{P}^{z}$ is the $(p \times p)$ covariance matrix of the error of the unknown input estimation and $\mathbf{P}^{z x}$ is the $(p \times n)$ covariance matrix of the cross-error of state and unknown input estimates $\left(\mathbf{P}^{z x}=\left(\mathbf{P}^{x z}\right)^{T}\right)$.

\section{APPLICATION OF KFUI}

To apply the KFUI, it is necessary to discretize the given system by (12), resulting in a system as presented in (13). The discretization is performed by the following transformations:

$$
\begin{aligned}
& \mathcal{F} \rightarrow \mathbf{F}, \mathcal{G} \rightarrow \mathbf{G}, \mathcal{E} \rightarrow \mathbf{E}, \mathcal{H} \rightarrow \mathbf{H}, \\
& \mathbf{x}(t) \rightarrow \mathbf{x}_{k}, \mathbf{x}_{k}=\left[\begin{array}{ll}
i_{p h_{k}} & v_{o_{k}}
\end{array}\right]^{T}, \\
& \tilde{\mathbf{x}}(t) \rightarrow \tilde{\mathbf{x}}_{k}, \tilde{\mathbf{x}}_{k}=\left[\begin{array}{ll}
\tilde{i}_{p h_{k}} & \tilde{v}_{o o_{k}}
\end{array}\right]^{T}, \\
& v_{p h}(t) \rightarrow v_{p h_{k}}=z_{k}, y(t) \rightarrow y_{k}=v_{o_{k}}, \\
& \tilde{v}_{p h}(t) \rightarrow \tilde{v}_{p h_{k}}=\tilde{z}_{k}, \tilde{y}(t) \rightarrow \tilde{y}_{k}=\tilde{v}_{o_{k}} .
\end{aligned}
$$




\subsection{Estimate of $i_{p h_{k}}$ and $v_{p h_{k}}$}

Knowing $V_{o}$ and by measuring the output voltage $v_{o_{k}}$, the disturbance $\tilde{v}_{o_{k}}$ is calculated from (9), that is:

$$
\tilde{v}_{o_{k}}=v_{o_{k}}-V o \text {. }
$$

Then, using the values of $\tilde{v}_{o_{k}}, \mathbf{F}, \mathbf{G}$ and $\mathbf{E}$ in (14) to (22) of the KFUI, the estimates $\hat{\vec{i}}_{p h_{k}}, \hat{\bar{v}}_{o_{k}}$ and $\hat{\hat{v}}_{p h_{k}}$ are obtained. Thus, $\hat{i}_{p h_{k}}, \hat{v}_{o_{k}}$ and $\hat{v}_{p h_{k}}$ are calculated from (24) to (26),

$$
\begin{gathered}
\hat{i}_{p h_{k}}=I_{p h}+\hat{\widetilde{i}}_{p h_{k}}, \\
\hat{v}_{o_{k}}=V_{o}+\hat{\widetilde{v}}_{O_{k}}, \\
\hat{v}_{p h_{k}}=V_{p h}+\hat{\widetilde{v}}_{p h_{k}} .
\end{gathered}
$$

\subsection{Estimate of irradiance}

Equation (27) represents (6) in discrete time,

$$
\begin{aligned}
G_{k}= & {\left[\frac{i_{p h_{k}}}{N_{P}}+i_{d k}+\frac{1}{R s h}\left(\frac{v_{p h_{k}}}{N_{S}}+\frac{i_{p h_{k}} R_{s}}{N_{P}}\right)\right] \times } \\
& \frac{G_{r}}{I_{s c}+\alpha_{I_{s} c}\left(T_{k}-T_{r}\right)} .
\end{aligned}
$$

An estimate $\hat{G}_{k}$ is obtained by substituting the estimates $\hat{v}_{p h_{k}}$ and $\hat{i}_{p h_{k}}$ in $(27)$.

\section{RESULTS AND DISCUSSION}

Some simulations of the circuit in Figure 2 together with KFUI were performed on MATLAB/SIMULINK software. Figure 3 shows a block diagram that briefly illustrates the simulations used.

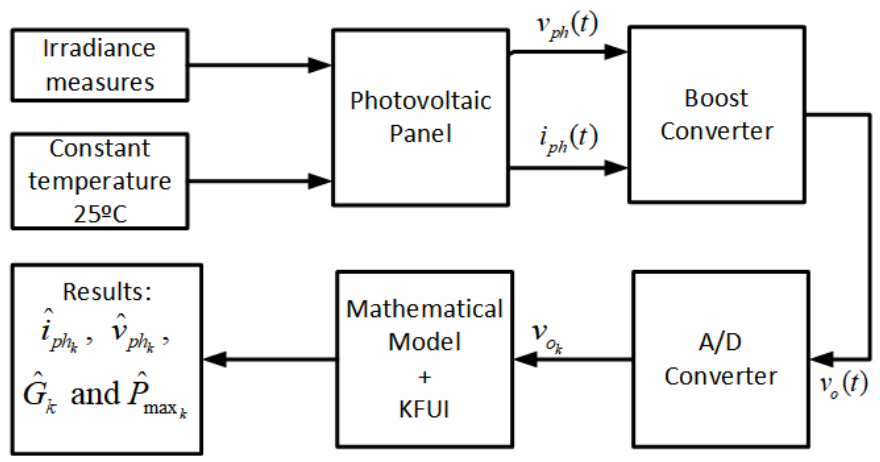

Figure 3. Block diagram of simulation

These simulations have the purpose of estimating the irradiance from the measurement of the output voltage of the dc-dc converter. The simulations were performed with irradiance measurements obtained in the city of Florianopolis - Brazil in June 2015 (National Institute for Space Research - INPE, 2018). Measurements have an interval of one minute.

A 1Soltech 1STH-215-P photovoltaic panel, which specifications are available in MATLAB's Specialized Power Systems Toolbox and are presented in Table 1 for standard irradiance of $1000 \mathrm{~W} / \mathrm{m}^{2}$, was used in simulations.

The simulated boost converter was designed according to (Erickson and Maksimović, 2001) and have the specifications shown in Table 2.
Table 1. Specifications of the 1STH-215-P solar panel

\begin{tabular}{lc}
\hline Maximum power & $213.15 \mathrm{~W}$ \\
Open circuit voltage & $36.30 \mathrm{~V}$ \\
Maximum power voltage & $29.00 \mathrm{~V}$ \\
Short-circuit current & $7.84 \mathrm{~A}$ \\
Maximum power current & $7.35 \mathrm{~A}$ \\
Cells per module & $6 \times 10$ \\
Diode ideality factor & 0.98 \\
Shunt resistance $R_{s h}$ & $313.40 \Omega$ \\
Series resistance $R_{S}$ & $0.39 \Omega$ \\
\hline
\end{tabular}

Table 2. Specifications of the boost converter

\begin{tabular}{ll}
\hline Quiescent input voltage $V_{p h}$ & $29.3 \mathrm{~V}$ \\
Quiescent output voltage $V_{\boldsymbol{O}}$ & $58 \mathrm{~V}$ \\
Duty cycle $D$ & 0.4948 \\
Inductance $L$ & $19.94 \mu \mathrm{H}$ \\
Capacitance $C$ & $36.34 \mu \mathrm{F}$ \\
Switching frequency & $50 \mathrm{kHz}$ \\
\hline
\end{tabular}

The used weighting matrices of KFUI were $\mathbf{V}=0.2030 \times 10^{-5}$ and $\mathbf{W}=\left[\begin{array}{cc}0.1807 \times 10^{-5} & 0 \\ 0 & 0.1807 \times 10^{-5}\end{array}\right]$.

\subsection{Case Studies}

A typical PV generation curve has a parabolic pattern and occurs on clear sky days (Miller et al., 2018). From the measurements made by National Institute for Space Research - INPE (2018), two days were analyzed with the following characteristics:

- Day 1: clear sky;

- Day 2: cloudy sky;

For each day, two cases were treated:

- Case 1: absence of noise in the output voltage of the converter;

- Case 2: presence of Gaussian noise with zero mean and standard deviation 1 in the measurement of $v_{o}(t)$;

Results were qualified by Normalized Root Mean Square Error (nRMSE), according to (28) (Scolari et al., 2017, 2018),

$$
n R M S E=\frac{1}{\bar{M}} \sqrt{\sum_{k=1}^{N} \frac{\left(M_{k}-\hat{M}_{k}\right)^{2}}{N}},
$$

where $N$ is the number of available measurements, $\bar{M}$ is the mean of the measurements, $M_{k}$ is the known $k$ th measurement and $\hat{M}_{k}$ is the estimated $k$ th of the irradiance obtained by KFUI.

\subsection{Day 1 - Clear sky}

On a possible clear sky day, which has a irradiance a typical photovoltaic curve, were obtained the estimates shown in Figures 4 to 6 . The resulting nRMSEs are presented in Table 3. In the two cases, the obtained nRMSEs has an average value of $3.65 \%$ and a maximum of $6.92 \%$.

In the work of Scolari et al. (2018) irradiance estimates performed on a real photovoltaic system presented nRM- 
SEs of $13.3 \%$ for a method based on all-sky camera and $13.6 \%$ for a Heliosat-2 method. Thus, the irradiance estimates $\hat{G}_{k}$ obtained by KFUI are compatible with these values found in literature.

Table 3. nRMSE for estimates of Day 1

\begin{tabular}{lccc}
\hline \multicolumn{4}{c}{ nRMSE[\%] } \\
\hline & $\hat{G}_{k}$ & $\hat{v}_{p h_{k}}$ & $\hat{i}_{p h_{k}}$ \\
\cline { 2 - 4 } Caso 1 & 0.93 & 5.66 & 0.65 \\
Caso 2 & 3.96 & 6.92 & 3.93 \\
\hline
\end{tabular}

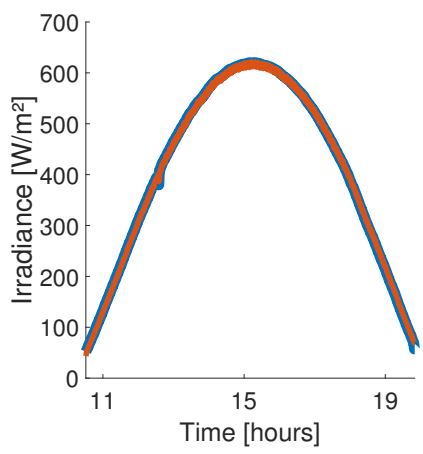

(a) Case 1

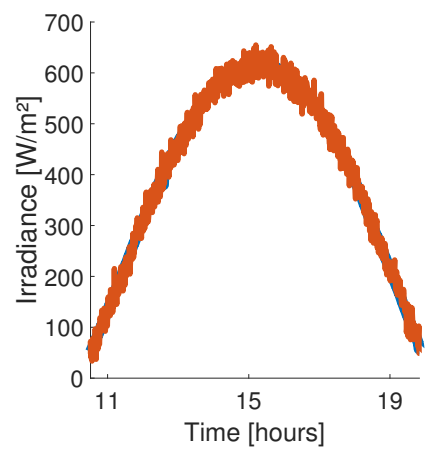

(b) Case 2

\section{- Real $=$ Estimate}

Figure 4. Irradiance estimate - Day 1

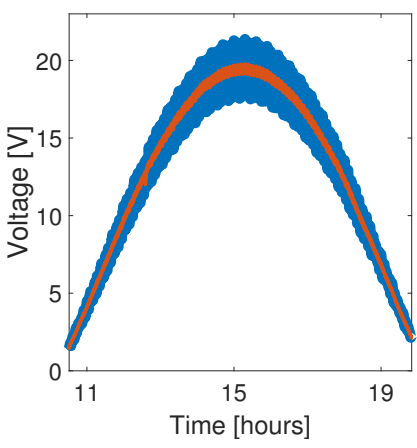

(a) Case 1

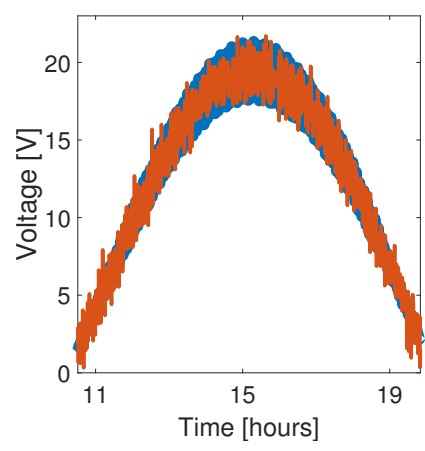

(b) Case 2

\section{- Real $=$ Estimate}

Figure 5. Estimate of the panel output voltage - Day 1

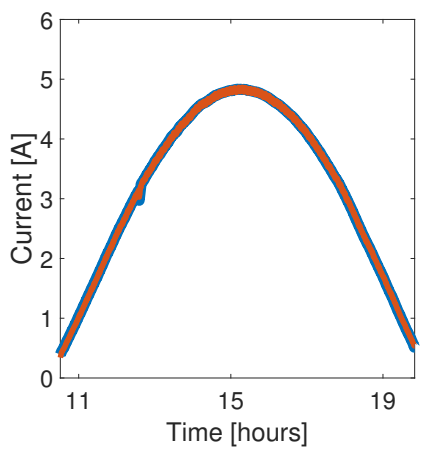

(a) Case 1

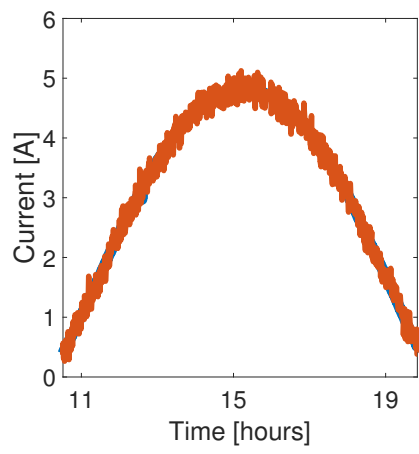

(b) Case 2

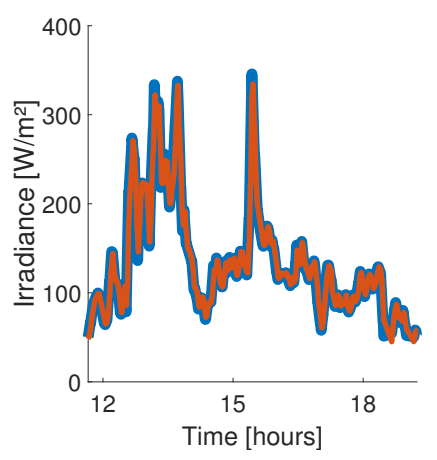

(a) Case 1

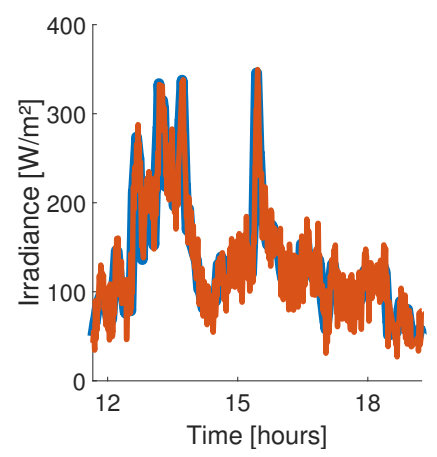

(b) Case 2
In the first step operation of boost converter, the capacitor, which has filtering attributes, is not associated with the voltage sensor. Thereby, directly measurement of $v_{p h}(t)$ has inherent degradation as shown in Figure 5. On the other hand, the KFUI estimation mitigates this switching component of $v_{p h}(t)$. Thus, the estimate $\hat{v}_{p h_{k}}$ is more reliable than direct measure $v_{p h_{k}}$ and consequently the nRMSE increases. Otherwise, the irradiance estimates would be bad, since $\hat{v}_{p h_{k}}$ is directly substituted in (27).

\subsection{Day 2: Cloudy sky}

The KFUI estimates for a possible cloudy day are shown in Figures 7 to 9 and the resulting nRMSEs are presented in Table 4 . The great amount of fluctuations in the irradiance curve increased the nRMSE values. However, the KFUI estimations follow the fluctuations of irradiance curve with a maximum nRMSE of $14.23 \%$.

Table 4. nRMSE for estimates of Day 2

\begin{tabular}{cccc}
\hline \multicolumn{4}{c}{ nRMSE[\%] } \\
\hline & $\hat{G}_{\boldsymbol{k}}$ & $\hat{v}_{\boldsymbol{p} \boldsymbol{h}_{\boldsymbol{k}}}$ & $\hat{\boldsymbol{i}}_{\boldsymbol{p} \boldsymbol{h}_{\boldsymbol{k}}}$ \\
\cline { 2 - 4 } Caso 1 & 8.32 & 5.72 & 4.55 \\
Caso 2 & 14.23 & 13.15 & 12.46
\end{tabular}

\section{Real $=$ Estimate}

Figure 7. Irradiance estimate - Day 2

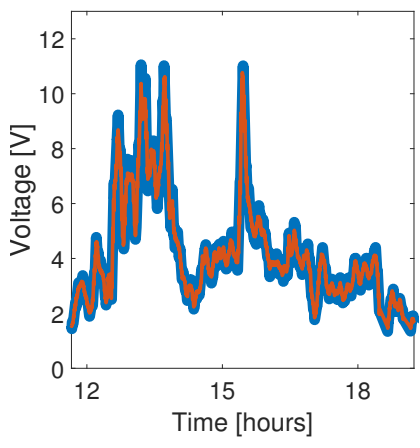

(a) Case 1

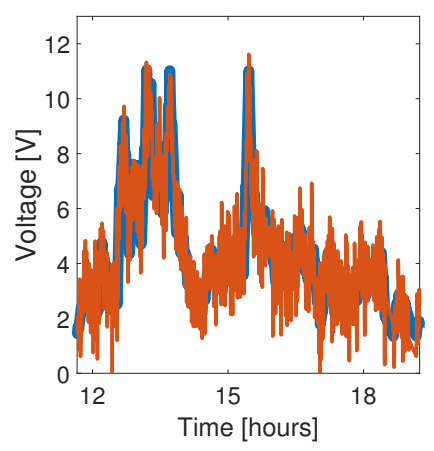

(b) Case 2
Real $=$ Estimate

Figure 6. Estimate of the panel output current - Day 1

Figure 8. Estimate of the panel output voltage - Day 2 


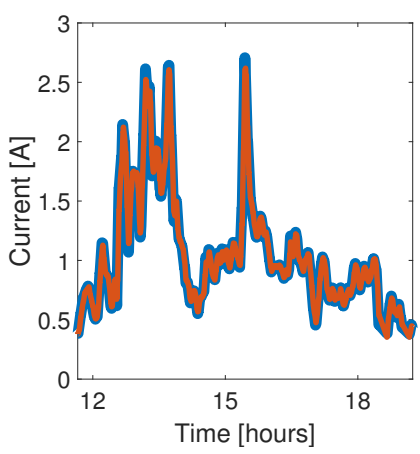

(a) Case 1

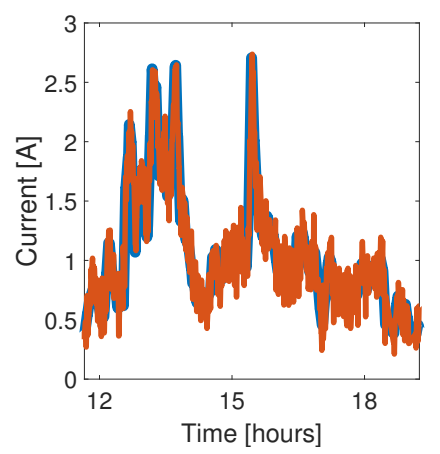

(b) Case 2

\section{Real $=$ Estimate}

Figure 9. Estimate of the panel output current - Day 2

These obtained estimations are compatible with results presented in Scolari et al. (2018), which nRMSEs on a cloudy day were $34.90 \%$ using all-sky camera and $47.90 \%$ using Heliosat-2.

\section{CONCLUSION}

In this paper, was presented a methodology of irradiance estimation in photovoltaic systems. To this end, a Kalman filter for systems subject to unknown input (KFUI) and mathematical models of the photovoltaic panel and the boost converter were used. A state space model was obtained in which the output voltage of the panel is considered an unknown input, making it possible to apply KFUI.

The simulated case studies shows that KFUI results are compatible with the values found in Scolari et al. (2018), where camera and satellite based methods were applied. The results of irradiance estimation provided nRMSEs between $0.93 \%$ and $14.23 \%$ for the worst case analyzed.

Additionally, the proposed methodology performs the estimation of the output voltage and the output current of the photovoltaic panel with nRMSEs of $5.66 \%-13.15 \%$ and $0.65 \%-12.46 \%$, respectively.

Thus, the irradiance estimation based on KFUI has good perspectives as it can be directly used in MPPT and several applications on control of photovoltaic systems.

\section{REFERENCES}

Chikh, A. and Chandra, A. (2015). An Optimal Maximum Power Point Tracking Algorithm for PV Systems with Climatic Parameters Estimation. IEEE Transactions on Sustainable Energy, 6(2), 644-652. doi:10.1109/TSTE. 2015.2403845 .

Darouach, M., Zasadzinski, M., Bassong, O., and Nowakowski, S. (1995). Kalman filtering with unknown inputs via optimal state estimation of singular systems. International Journal of Systems Science, 26(10), 20152028. doi:10.1080/00207729508929152.

Erickson, R.W. and Maksimović, D. (2001). Fundamentals of Power Electronics. Springer US, Boston, MA. doi: $10.1007 / \mathrm{b} 100747$.
Martins, F.R. and Pereira, E.B. (2011). Estudo comparativo da confiabilidade de estimativas de irradiação solar para o sudeste brasileiro obtidas a partir de dados de satélite e por interpolação/extrapolação de dados de superfície. Revista Brasileira de Geofisica, 29(2), 265276. doi:10.1590/S0102-261X2011000200005.

Miller, S.D., Rogers, M.A., Haynes, J.M., Sengupta, M., and Heidinger, A.K. (2018). Short-term solar irradiance forecasting via satellite/model coupling. Solar Energy, 168(October), 102-117. doi:10.1016/j.solener.2017.11. 049 .

Mohamed, A. and Mohammed, O. (2013). Real-time energy management scheme for hybrid renewable energy systems in smart grid applications. Electric Power Systems Research, 96, 133-143. doi:10.1016/j.epsr.2012. 10.015 .

Moshksar, E. and Ghanbari, T. (2018). A model-based algorithm for maximum power point tracking of $\mathrm{PV}$ systems using exact analytical solution of single-diode equivalent model. Solar Energy, 162(May 2017), 117131. doi:10.1016/j.solener.2017.12.054.

National Institute for Space Research - INPE (2018). National system of ambiental data organization - SONDA. URL http://sonda.ccst.inpe.br/.

Nguyen, X.H. and Nguyen, M.P. (2015). Mathematical modeling of photovoltaic cell/module/arrays with tags in Matlab/Simulink. Environmental Systems Research, 4(1), 24. doi:10.1186/s40068-015-0047-9.

Pereira, E.B., Martins, F.R., Gonçalves, André Rodrigues Costa, R.S., Lima, Francisco J. Lopes de Rüther, R., de Abreu, S.L., Tiepolo, G.M., Pereira, S.V., and de Souza, J.G. (2017). Atlas Brasileiro de Energia Solar. INPE, São José dos Campos, 2 edition. doi: 978-85-17-00030-0.

Scolari, E., Sossan, F., Haure-Touzé, M., and Paolone, M. (2018). Local estimation of the global horizontal irradiance using an all-sky camera. Solar Energy, 173, 1225-1235. doi:10.1016/j.solener.2018.08.042.

Scolari, E., Sossan, F., and Paolone, M. (2017). Photovoltaic-Model-Based Solar Irradiance Estimators: Performance Comparison and Application to Maximum Power Forecasting. IEEE Transactions on Sustainable Energy, 9(1), 35-44. doi:10.1109/TSTE.2017.2714690.

Tian, H., Mancilla-David, F., Ellis, K., Muljadi, E., and Jenkins, P. (2012). A cell-to-module-to-array detailed model for photovoltaic panels. Solar Energy, 86(9), 2695-2706. doi:10.1016/j.solener.2012.06.004. 